\title{
Nuclear Astrophysics at LUNA: recent results
}

\author{
Alessandra Guglielmetti for the LUNA collaboration ${ }^{1}$ \\ Università degli Studi di Milano and INFN Milano \\ Via Celoria 16, I-20133 Milano, Italy \\ E-mail: alessandra.guglielmetti@mi.infn.it
}

\begin{abstract}
At temperatures typical of astrophysical environments, nuclear cross sections are extremely small and experimental measurements in a laboratory at the Earth's surface are hampered by the cosmic background. The LUNA collaboration has exploited the unique features of background reduction offered by the rock overburden above the LNGS underground laboratory in order to study very important hydrogen-burning reactions at their relevant astrophysical energies. After a general introduction on the LUNA experiment, this paper reports on the most important results obtained so far and their astrophysical consequences. An overview on the future possibilities of this experiment through a consistent upgrade of the available experimental apparatus is also given.
\end{abstract}

VI European Summer School on Experimental Nuclear Astrophysics Acireale Italy

September 18-27, 2011

1 Speaker 


\section{Introduction}

Energy generation and element production in stars are accomplished by means of thermonuclear fusion reactions $[1,2]$, which start from the most abundant and lightest element, hydrogen, and gradually synthesize heavier elements. The first nuclear fusion process is the socalled hydrogen burning which has the net result of transforming 4 protons into a ${ }^{4} \mathrm{He}$ nucleus with an energy release of about $27 \mathrm{MeV}$. Hydrogen burning coincides with the longest stage of a star's life (also know as its main sequence phase) and is responsible for the prodigious luminosity of the star itself. It mainly proceeds either through the p-p chain or through the more efficient $\mathrm{CNO}$ cycle. The latter and successive cycles such as the $\mathrm{NeNa}$ and $\mathrm{MgAl}$ become important for second generation stars whose central temperatures and masses are higher than those of our Sun and whose evolution stage is such that the necessary seeds for these reactions are already present. Due to the higher Coulomb barriers, these cycles are relatively unimportant for energy generation but are essential for the nucleosynthesis of elements with mass number higher than 20.

All these fusion reactions occur in a very well defined energy range, the so-called Gamow peak, which arises from the convolution of the energy distribution of nuclei in the stellar plasma and the tunneling probability through the Coulomb barrier between the interacting nuclei. In a non-degenerate, non-relativistic stellar plasma, the former is given by the Maxwell-Boltzmann distribution, with a maximum for $\mathrm{E}=\mathrm{kT}$ (where $\mathrm{T}$ is the star temperature and $\mathrm{k}$ the MaxwellBoltzmann constant) and then decreases exponentially for increasing energy, while the latter decreases exponentially for decreasing energy. For example, for a central temperature of $1.5 \cdot$ $10^{7} \mathrm{~K}$, as in our Sun, the maximum of the Maxwell-Boltzmann distribution occurs at about 1 $\mathrm{keV}$, while the Coulomb barrier for most reactions in either the p-p chain or the CNO cycle is between 0.5 and $2 \mathrm{MeV}$. The Gamow peak for the same reactions is below $30 \mathrm{keV}$ and as a result reaction cross sections can be extremely low, down to the femto-barn level in "the bestcase scenario" due to the already mentioned exponential drop of the tunneling probability with decreasing energy. It follows that a direct investigation of thermonuclear reactions at or near their Gamow energy is often beyond technical capabilities as the signal-to-noise ratio is severely dominated by any source of unwanted background. In a laboratory at the Earth's surface, the greatest contribution to the background typically arises from the interaction of cosmic rays with the material in the detection setup. Reactions cross-sections are therefore extrapolated from data taken at higher energies, albeit with significant uncertainties remaining at the lowest energies of astrophysical interest. The extrapolation procedure is typically carried out on the so-called astrophysical $\mathrm{S}(\mathrm{E})$-factor defined by:

$\sigma(\mathrm{E})=\mathrm{E}^{-1} \mathrm{~S}(\mathrm{E}) \exp (-2 \pi \eta)$

where $\eta$ is the Sommerfeld parameter given by:

$\eta=(31.29 / 2 \pi) Z_{1} Z_{2}(\mu / E) 1 / 2$ 
with $Z_{1}$ and $Z_{2}$ integral charges of the interacting nuclei; $\mu$ the reduced mass; and $E$ the centerof-mass interaction energy in $\mathrm{keV}$. The astrophysical S-factor contains the purely nuclear properties of the interaction and is generally a more smoothly varying function of energy than the cross section. However, the existence of narrow resonances in or near the Gamow energy region, the tails of broad resonances and/or sub-threshold states, as well as a change in the reaction mechanism at ultra-low energies, can all translate in non-negligible contributions to the reaction cross section (or equivalently the astrophysical factor) thus rendering the extrapolation procedure extremely uncertain.

An alternative solution to the extrapolation procedure requires a drastic reduction of any unwanted background so as to optimize the signal-to-noise ratio. This can often be achieved by carrying out the measurement in deep underground laboratories, as also underlined in the contribution of M.L. Aliotta at this Summer School.

\section{Why underground: the LUNA project}

The LUNA (Laboratory for Underground Nuclear Astrophysics) collaboration has exploited the low-background environment of the underground laboratory under the Gran Sasso Mountain in Italy (LNGS) to perform direct measurements at the relevant astrophysical energies. The rock overburden of about $1400 \mathrm{~m}$ ( $3800 \mathrm{~m}$ water equivalent) reduces the muon component of the cosmic background by a factor of $10^{6}$; the neutron component by a factor of $10^{3}$; and the gamma component by a factor of 10 with respect to a laboratory on the Earth's surface. As a result, the gamma background above $3 \mathrm{MeV}$ in an HPGe detector placed underground at LNGS is reduced by a factor of $\sim 2500$ with respect to the same detector placed over-ground. In addition, going underground enhances the effect of passive shielding particularly for lower energy gammas where the background is dominated by environmental radioactivity. Indeed, a passive shield can be built around the detector also in a laboratory at the Earth's surface. However, the shielding efficiency cannot be increased by further adding any more shield since the cosmic muons would interact with the added material- creating more background. This problem is of course dramatically reduced in an underground laboratory.

The LUNA collaboration has installed two accelerators underground: a compact $50 \mathrm{kV}$ "home-made" machine [3] and a commercial $400 \mathrm{kV}$ one [4]. Common features of the two are the intense beam currents achievable, the long-term stability, and the precise energy determination. The first two features are essential to maximize the reaction rate, while the third is important in view of the exponential energy dependence of the cross section. With the first machine, operating between 1992 and 2001, two key reactions of the p-p chain were studied at the solar Gamow peak energies: the ${ }^{3} \mathrm{He}\left({ }^{3} \mathrm{He}, 2 \mathrm{p}\right)^{4} \mathrm{He}$ [5] and $\mathrm{d}(\mathrm{p}, \gamma)^{3} \mathrm{He}$ [6]. In addition, the screening effect, i.e. lowering of the Coulomb barrier for a nucleus surrounded by electrons with respect to a bare one, was investigated through the $\mathrm{d}\left({ }^{3} \mathrm{He}, \mathrm{p}\right)^{4} \mathrm{He}$ reaction [7]. The $400 \mathrm{kV}$ machine started operations in the year 2000 and is still operating. 


\section{Results}

Among the main results obtained so far with the $400 \mathrm{kV}$ machine are the fusion cross sections of the ${ }^{14} \mathrm{~N}(\mathrm{p}, \gamma){ }^{15} \mathrm{O}$ and ${ }^{3} \mathrm{He}\left({ }^{4} \mathrm{He}, \gamma\right){ }^{7} \mathrm{Be}$ reactions. More recently, the LUNA collaboration has been engaged in measuring two other important $(\mathrm{p}, \gamma)$ fusion reactions, namely the ${ }^{25} \mathrm{Mg}(\mathrm{p}, \gamma){ }^{26} \mathrm{Al}$ and the ${ }^{15} \mathrm{~N}(\mathrm{p}, \gamma){ }^{16} \mathrm{O}$ reaction: in this paper, I will report only on the first one.

\subsection{The ${ }^{14} \mathrm{~N}(\mathrm{p}, \gamma){ }^{15} \mathrm{O}$ reaction}

The ${ }^{14} \mathrm{~N}(\mathrm{p}, \gamma){ }^{15} \mathrm{O}$ reaction is the slowest reaction in $\mathrm{CNO}$ cycle and it thus controls the cycle's energy-production rate as well as the ${ }^{13} \mathrm{~N}$ and ${ }^{15} \mathrm{O}$ solar neutrino flux that depends almost linearly on the reaction rate. The reaction also affects the age of globular clusters, i.e. conglomerates of $10^{4}$ to $10^{6}$ gravitationally bound stars among the oldest stars in our galaxy. The luminosity of the turnoff point in the Hertzsprung-Russell diagram of a globular cluster, namely the point at which the main sequence turns toward cooler and brighter stars, is used to determine the age of the cluster and to derive a lower limit on the age of the universe [8]. For a star at the turnoff point hydrogen shell burning is powered by the CNO cycle and therefore the ${ }^{14} \mathrm{~N}(\mathrm{p}, \gamma){ }^{15} \mathrm{O}$ cross section plays a crucial role in the age determination: the higher the cross section, the younger the globular cluster's age for a given turn-off luminosity.

In the first phase of the LUNA study, data were obtained down to $\mathrm{E}_{\mathrm{CM}}=119 \mathrm{keV}$ using solid TiN targets and a high efficiency $\mathrm{HPGe}$ detector. Five different radiative-capture transitions that contribute to the ${ }^{14} \mathrm{~N}(\mathrm{p}, \gamma){ }^{15} \mathrm{O}$ cross section at low energy were identified and measured.

In the second phase of the experiment the total cross section was then studied down to very low energies $\left(E_{\mathrm{CM}}=70 \mathrm{keV}\right)$ using a $4 \pi$ BGO summing detector placed around a windowless gas target filled with nitrogen at a pressure of 1 mbar. At the lowest energy of 70 $\mathrm{keV}$, a cross section of $0.24 \mathrm{pb}$ was measured, corresponding to an event rate of 11 counts per day. The results obtained first with the germanium detector $[9,10]$ and then with the BGO setup [11] were approximately a factor of 2 lower than the existing extrapolation [12] from previous data [13] at very low energy. As a consequence, the CNO neutrino yield in the Sun is reduced by about a factor of 2, and the age of the globular clusters is increased by 0.7 to 1 billion years, thus raising the age of the Universe to 14 billion years [14].

The main conclusion from the LUNA data has been confirmed by an independent study at higher energy [15]. However, there is a $15 \%$ discrepancy between the total S-factors extrapolated by the two experiments at the Gamow peak of the Sun. This difference arises from the extrapolation of the capture to the ground state in ${ }^{15} \mathrm{O}$, a transition strongly affected by interference effects between several resonances and the direct-capture mechanism. In order to obtain more precise data for the capture into the ground-state, a third phase of the ${ }^{14} \mathrm{~N}(\mathrm{p}, \gamma){ }^{15} \mathrm{O}$ study was performed with a clover detector consisting of 4 closely packed individual HPGe crystals and a surrounding BGO detector for anti-Compton shielding, thus minimizing the summing correction effects. Measurements were taken in the beam-energy region immediately above the $259 \mathrm{keV}$ resonance, where precise data effectively constrain a fit for the ground-state transition in the R-matrix framework. The total error on the S-factor was reduced to $8 \%$ [16]. 
This is significant because, having finally solved the solar neutrino problem, we are now facing the problem of solar composition. There is a conflict between helioseismology and the new metal abundances that emerged from improved modeling of the photosphere [17]. Thanks to the relatively small error, soon it will be possible to measure the metallicity of the core of the Sun (i.e., the elements other than hydrogen and helium) by comparing the detected $\mathrm{CNO}$ neutrino flux with the predicted flux.

\subsection{The ${ }^{3} \mathrm{He}\left({ }^{4} \mathrm{He}, \gamma\right){ }^{7} \mathrm{Be}$ reaction}

The ${ }^{3} \mathrm{He}\left({ }^{4} \mathrm{He}, \gamma\right){ }^{7} \mathrm{Be}$ reaction provides the onset for the ${ }^{7} \mathrm{Be}$ and ${ }^{8} \mathrm{~B}$ branches of the $\mathrm{p}$-p chain from where the ${ }^{7} \mathrm{Be}$ and ${ }^{8} \mathrm{~B}$ neutrinos are generated. Solar neutrino fluxes depend on both astrophysical and nuclear physics inputs: in the case of ${ }^{7} \mathrm{Be}$ and ${ }^{8} \mathrm{~B}$ neutrinos, one of the most important parameters is the cross section of the ${ }^{3} \mathrm{He}\left({ }^{4} \mathrm{He}, \gamma\right)^{7} \mathrm{Be}$ reaction. After the successful measurements of the ${ }^{8} \mathrm{~B}$ neutrino flux with a $3.5 \%$ uncertainty performed by SNO [18] and SuperKamiokande [19] and the very recent result on the ${ }^{7} \mathrm{Be}$ neutrino flux from the Borexino experiment [20], a comparable uncertainty on the ${ }^{3} \mathrm{He}\left({ }^{4} \mathrm{He}, \gamma\right){ }^{7} \mathrm{Be}$ cross section was required before a fuller understanding of the physical and chemical properties of the Sun's interior could be arrived at. The ${ }^{3} \mathrm{He}\left({ }^{4} \mathrm{He}, \gamma\right){ }^{7} \mathrm{Be}$ reaction also plays a critical role in the Big-Bang nucleosynthesis $(\mathrm{BBN})$, being the main source of ${ }^{7} \mathrm{Li}$ production. The predicted abundance of such an isotope is a factor of 2-3 higher than the observed one. Even if it is unlikely that the explanation of this discrepancy could come entirely from the ${ }^{3} \mathrm{He}\left({ }^{4} \mathrm{He}, \gamma\right){ }^{7} \mathrm{Be}$ cross section, a better knowledge of its value represents a necessary pre-requisite for the search of alternative solutions to the ${ }^{7} \mathrm{Li}$ problem.

Over last forty years, the ${ }^{3} \mathrm{He}\left({ }^{4} \mathrm{He}, \gamma\right){ }^{7} \mathrm{Be}$ reaction has been measured using two different approaches: either by detection of the direct $\alpha$-capture $\gamma$-rays (prompt $\gamma$ method) or by detection of the ${ }^{7} \mathrm{Be}$ radioactivity through the electron capture of ${ }^{7} \mathrm{Be}$ into ${ }^{7} \mathrm{Li}$ and its subsequent deexcitation from the first excited to the ground state (activation method). Results obtained with the two techniques show, on average, a discrepancy of about $11 \%$ which is at the origin of the large uncertainty on the ${ }^{3} \mathrm{He}\left({ }^{4} \mathrm{He}, \gamma\right){ }^{7} \mathrm{Be}$ cross section [21].

To solve this problem, the LUNA collaboration planned and performed an experiment with both techniques at the same time in order to minimize systematical uncertainties. A recirculating ${ }^{3} \mathrm{He}$ extended windowless gas target was coupled to a $137 \%$ efficiency HPGe detector in close geometry (100\% refers to a 3 inches diameter and 3 inches length cylindrical $\mathrm{NaI}$ detector). The intense ${ }^{4} \mathrm{He}$ beam of the LUNA $400 \mathrm{kV}$ machine was focused onto a removable copper beam-stop, which served both as the end cap of a calorimeter (to measure the beam current) and as a catcher for the ${ }^{7} \mathrm{Be}$ nuclei allowing for a delayed off-line measurement of their activity. Since the reaction Q-value is about $1.5 \mathrm{MeV}$, the prompt $\gamma$-rays are in the region of natural background and a massive shielding $\left(0.3 \mathrm{~m}^{3}\right)$ was therefore built around the target chamber and the HPGe detector. This consisted of several layers of lead bricks and an inner layer of Oxygen Free High Conductivity (OFHC) copper bricks. In addition, the target chamber and the calorimeter were constructed using OFHC copper and no welding materials were used. The setup was enclosed in an anti-radon envelope consisting in a Plexiglas box flushed with $\mathrm{N}_{2}$ gas to avoid ${ }^{222} \mathrm{Rn}$ accumulation. As already mentioned, the efficiency of the shielding could be 
increased thanks to the underground location and a background suppression of five orders of magnitude with respect to a background spectrum measured underground with no shielding was obtained for $\gamma$-rays below $2 \mathrm{MeV}$ [22]. The typical $\alpha$-beam current was high (about $250 \mu \mathrm{A}$ ), thus causing a reduction of the target density due to the so-called beam-heating effect. This was measured through the detection of the beam particles elastically scattered on the target atoms [23]. In order to reduce the systematic error coming from the uncertainty on the angular distribution, a lead collimator was positioned inside the target chamber to collect mostly $\gamma$-rays emitted at $55^{\circ}$, where the contribution of the second Legendre polynomial vanishes. After each run, the calorimeter cap was dismounted and its activity counted off-line with a $125 \%$ HPGe detector (see before for the reference efficiency data), shielded by $15 \mathrm{~cm}$ of lead and $10 \mathrm{~cm}$ of copper on each side and placed in the low activity laboratory of LNGS [24].

The experiment was divided into two phases: in the first one, the cross section was measured at $\mathrm{E}_{\mathrm{CM}}=127$ and $148 \mathrm{keV}$ with the activation technique only [25], in the second one, both techniques were used to measure the cross section at $\mathrm{E}=93,106$ and $170 \mathrm{keV}[26,27]$. A comparison of the results obtained with the two techniques was therefore possible and reduced significantly the systematic uncertainties. The cross sections measured with the two methods turned out to be consistent within the quoted experimental errors [26, 27]. The energy region covered by LUNA is above the Gamow peak for the Sun but well within the Gamow peak for the BBN, as shown in Figure 1.

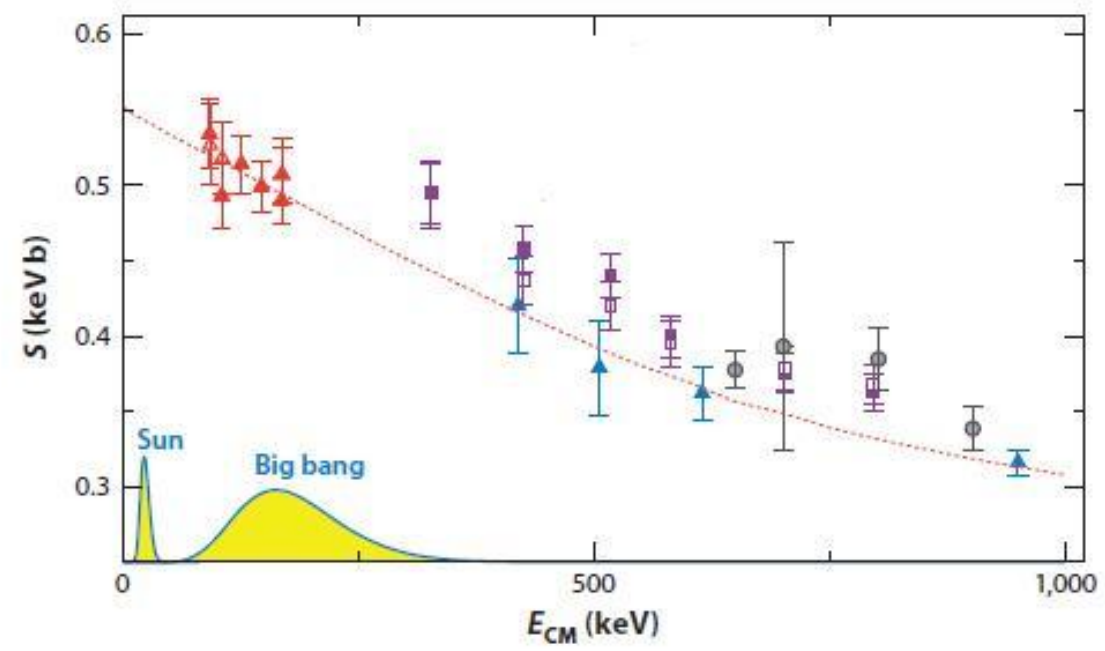

Figure 1: Astrophysical S-factor of the ${ }^{3} \mathrm{He}\left({ }^{4} \mathrm{He}, \gamma\right)^{7} \mathrm{Be}$ reaction: the LUNA activation results are shown as filled red triangles [25] while the prompt- $\gamma$ ones are shown as empty red triangles [26,27].The Gamow peak regions for the Sun and Big bang are shown in yellow. All the other data points refer to recent measurements of the same reaction [28,29,30].

Our precise results clearly rule out the ${ }^{3} \mathrm{He}\left({ }^{4} \mathrm{He}, \gamma\right)^{7} \mathrm{Be}$ cross section as a possible source of the discrepancy between the predicted primordial ${ }^{7} \mathrm{Li}$ abundance and its much lower observed value. The LUNA results and few other high precision measurements $[28,29,30]$ were included in the recent compilation "Solar fusion II" [31] to derive the best astrophysical S-factor for the ${ }^{3} \mathrm{He}\left({ }^{4} \mathrm{He}, \gamma\right){ }^{7} \mathrm{Be}$ reaction. The value obtained allows for a uncertainty reduction in the solar 
neutrino fluxes from $7.5 \%$ to $4.3 \%$ in the case of ${ }^{8} \mathrm{~B}$ and from $8 \%$ to $4.5 \%$ in the case of ${ }^{7} \mathrm{Be}$ [32].

\subsection{The ${ }^{25} \mathrm{Mg}(\mathrm{p}, \gamma)^{26} \mathrm{Al}$ reaction}

This is the slowest reaction of the $\mathrm{Mg}-\mathrm{Al}$ cycle. The $\beta^{+}$decay of ${ }^{26} \mathrm{Al}\left(\mathrm{T}_{1 / 2}=7 \cdot 10^{5} \mathrm{y}\right)$ populates the $1.8 \mathrm{MeV}$ excited state of ${ }^{26} \mathrm{Mg}$ which then decays to the ground state by $\gamma$ emission. Observations from satellites $[33,34]$ have determined an amount of ${ }^{26} \mathrm{Al}$ in our galaxy of about 6 solar masses through the detection of this $\gamma$-ray line. Moreover, evidences for an ${ }^{26} \mathrm{Al}$ excess in the early solar system was found in calcium aluminum inclusions showing a significant correlation of ${ }^{26} \mathrm{Mg}$ (extinct ${ }^{26} \mathrm{Al}$ ) and ${ }^{27} \mathrm{Al}$ [35]. While the former observation gives evidence that ${ }^{26} \mathrm{Al}$ nucleosynthesis is still active on a large scale, the latter suggests that ${ }^{26} \mathrm{Mg}$ also was produced at the time of the condensation of the solar-system material, about 4.6 billion years ago. ${ }^{26} \mathrm{Al}$ is mainly produced via the ${ }^{25} \mathrm{Mg}(\mathrm{p}, \gamma){ }^{26} \mathrm{Al}$ reaction which is active in the hydrogen burning shell of off-main sequence stars of any mass and in the carbon burning regions of massive stars. Different astrophysical environments are therefore responsible for ${ }^{26} \mathrm{Al}$ nucleosynthesis and the reaction cross section should be precisely studied in a wide energy range since each environment is characterized by a temperature range corresponding to a specific energy window. In particular, the ${ }^{25} \mathrm{Mg}(\mathrm{p}, \gamma)^{26} \mathrm{Al}$ reaction is dominated by narrow resonances, giving rise to a rather complex decay scheme of $\gamma$-ray cascades. Before LUNA, the strengths of these resonances were experimentally studied only down to $190 \mathrm{keV}$, in some cases with large discrepancies among the results obtained with different techniques. Indeed, the reaction can be studied by detecting the prompt gamma rays or by counting the ${ }^{26} \mathrm{Al}$ nuclei with accelerator mass spectroscopy (AMS). At LUNA, several resonances were investigated with different and complementary techniques: a low-efficiency and high-resolution HPGe detector was used for the higher energy resonances (304 and $190 \mathrm{keV}$ ); a low-resolution but highefficiency BGO detector was used also for the lower energy resonances (304, 190, 130 and 93 $\mathrm{keV}$ ); and the AMS technique was used for the $304 \mathrm{keV}$ resonance at the CIRCE facility in Caserta (Italy) [36] on the LUNA proton-irradiated Mg targets. The $304 \mathrm{keV}$ resonance was measured with the three techniques in order to understand possible systematic uncertainties and compare with previous results. A major effort was devoted to the study of the target stoichiometry since this is a critical parameter for determining the reaction rate. Both natural $\mathrm{Mg}$ targets (of well known isotopic composition) and enriched ${ }^{25} \mathrm{Mg}$ targets were used. The former for determining the resonance strengths of relatively high energy resonances of $(\mathrm{p}, \gamma)$ reactions on ${ }^{24,25,26} \mathrm{Mg}$ isotopes, while the latter only for the ${ }^{25} \mathrm{Mg}(\mathrm{p}, \gamma){ }^{26} \mathrm{Al}$ reaction. The results obtained with the natural targets give normalization for those obtained with the enriched targets, also in the case of lower energy resonances. For the higher energy resonances, the following results were obtained [37]: for the $304 \mathrm{keV}$ resonance of ${ }^{25} \mathrm{Mg}(\mathrm{p}, \gamma){ }^{26} \mathrm{Al}$ reaction, measured with three different techniques (HPGe, BGO and AMS), a very good agreement for the resonance strength was found among the different approaches. A comparison of the LUNA results with data in the literature shows a strong disagreement only with the result of Arazi et al. [38] obtained with the AMS technique. The NACRE evaluation [12] combined with the LUNA result yields an improved uncertainty of $4 \%$. For the $214 \mathrm{keV}$ resonance of the ${ }^{24} \mathrm{Mg}(\mathrm{p}, \gamma)^{25} \mathrm{Al}$ 
reaction, the LUNA resonance strength is lower by more than $10 \%$ with respect to Powell et al. [39]. However, a strong direct capture component dominates the resonance contribution and a re-analysis of the reaction at astrophysical energies using R-matrix formalism with improved $\gamma$ width knowledge is worthwhile. For the ${ }^{26} \mathrm{Mg}(\mathrm{p}, \gamma)^{27} \mathrm{Al} 326 \mathrm{keV}$ resonance, an apparent discrepancy in the literature [12] was solved and the resonance strength was measured with a higher accuracy.

The $190 \mathrm{keV}$ resonance of the ${ }^{25} \mathrm{Mg}(\mathrm{p}, \gamma)^{26} \mathrm{Al}$ reaction was measured with HPGe and BGO detectors. The results obtained for the resonance strength with the two techniques [40] are in excellent agreement among themselves while in disagreement with the results of [41]. Possible explanations might be the influence of an angular distribution or an incomplete summing-out correction in the $0 \circ$ measurement of [41]. Moreover, the primary $\gamma$-ray branching ratios were determined from the measurements with the HPGe detector and the probability of populating the ground state of ${ }^{26} \mathrm{Al}$ was obtained from the full decay scheme of this resonance including all known secondary transitions. Figure 2 shows an HPGe $\gamma$-ray spectrum obtained summing several runs: the background reduction given by the underground measurement allowed us to determine several primary and secondary transitions populated in the reaction.

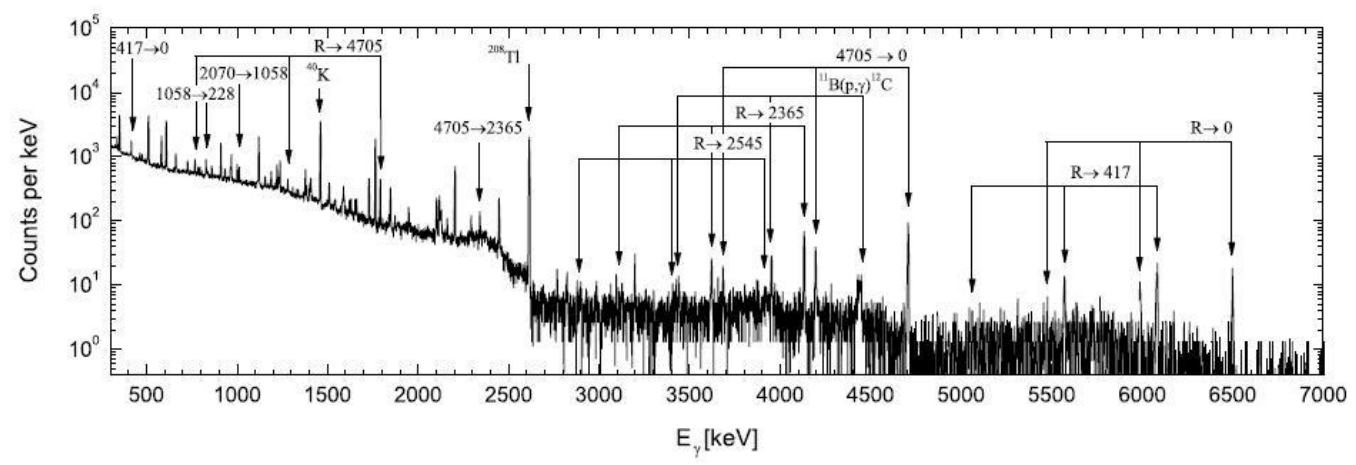

Figure 2: The HPGe $\gamma$-ray spectrum taken at the $190 \mathrm{keV}{ }^{25} \mathrm{Mg}(p, \gamma)^{26} \mathrm{Al}$ resonance: the most prominent primary transitions, important secondary transitions and some $\gamma$-ray background lines are shown.

In spite of the large sum peak efficiency of the BGO detector and the high beam intensity, only the $93 \mathrm{keV}$ resonance could be clearly observed below $\mathrm{E}=190 \mathrm{keV}$ [40]. Figure 3 shows the BGO $\gamma$-ray total sum spectrum obtained at this energy: the presence of a peak at the expected energy is well evident. 


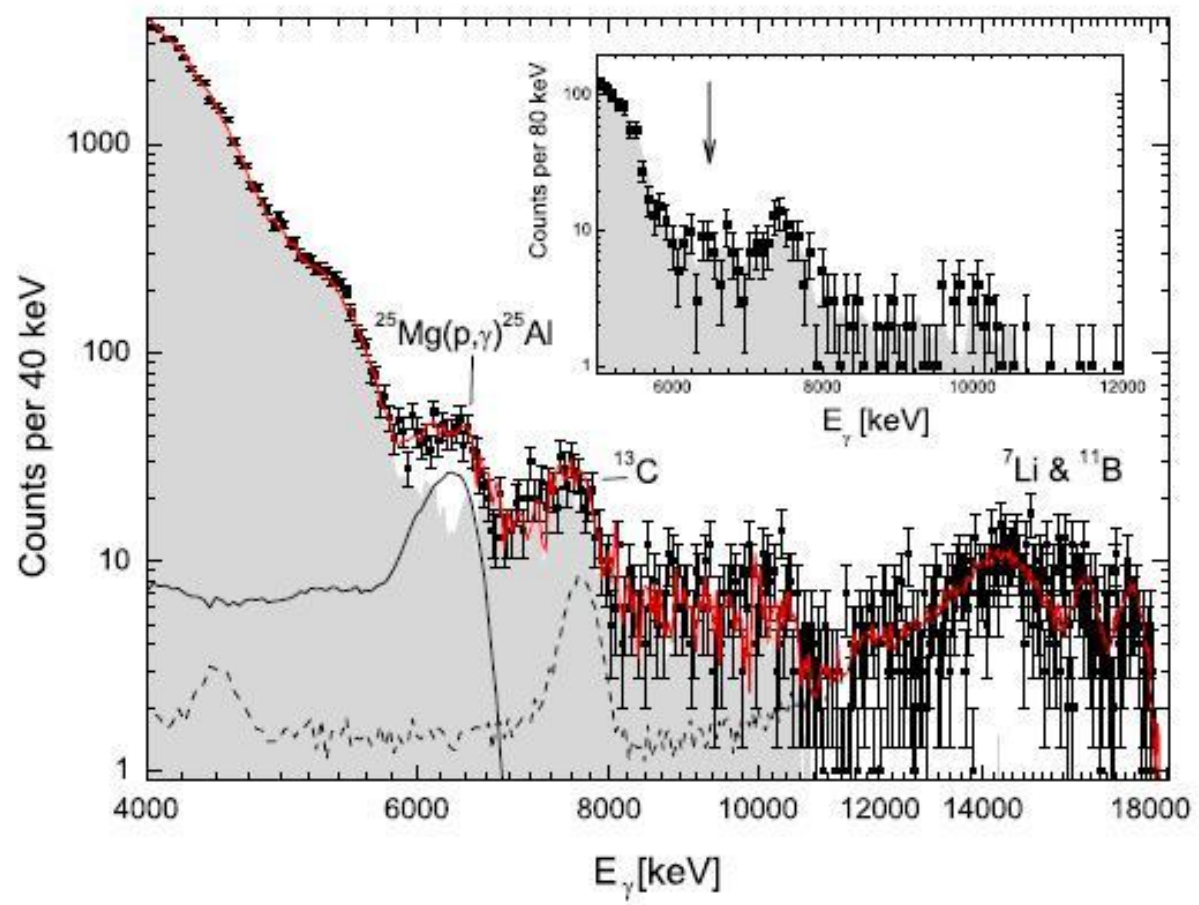

Figure 3: The BGO $\gamma$-ray total sum spectrum on the $93 \mathrm{keV}{ }^{25} \mathrm{Mg}(p, \gamma)^{26} \mathrm{Al}$ resonance. The shaded area, thin solid line, and solid red line represent the environmental background, the $25 \mathrm{Mg}(\mathrm{p}, \gamma) 26 \mathrm{Al}$ GEANT4 simulation, and the total yield fit including background and simulation, respectively. The contributions from contaminant reactions (dashed line from GEANT4 simulations) are labeled. The insert shows the background at $E=86.5 \mathrm{keV}$, i.e. below the resonance. The region of the sum peak is indicated by an arrow and its absence is well evident.

In addition, for the $130 \mathrm{keV}$ resonance an upper limit was obtained [40] while all other low-energy resonances are too weak to be studied with this experimental setup. The $93 \mathrm{keV}$ resonance was directly observed for the first time and the corresponding resonance strength is to our knowledge the lowest ever directly measured. The result corrected for the screening effect is within the quoted

uncertainty in agreement with the NACRE value [12] determined from indirect measurements. The ground state population of ${ }^{26} \mathrm{Al}$ was determined also for this resonance from the full decay scheme. The first direct study of the $93 \mathrm{keV}$ resonance largely reduces the uncertainty in the reaction rate.

\section{On-going measurements}

Presently the LUNA collaboration is measuring the ${ }^{17} \mathrm{O}(\mathrm{p}, \gamma){ }^{18} \mathrm{~F}$ and the ${ }^{2} \mathrm{H}(\alpha, \gamma){ }^{6} \mathrm{Li}$ reactions. In this paper I will report only on the latter since the former is the object of the contribution of D. Scott to this Summer School.

The ${ }^{2} \mathrm{H}(\alpha, \gamma)^{6} \mathrm{Li}$ reaction is fundamental in the BBN and determines the amount of primordial ${ }^{6} \mathrm{Li}$ in the universe. Recently, ${ }^{6} \mathrm{Li}$ has been detected in a number of metal poor stars $[42,43]$ and its quantity has been found to be higher than expected from BBN by 2-3 orders of magnitude [44]. Direct measurements of this reaction have been performed only down to $1 \mathrm{MeV}$ 
and around the $711 \mathrm{keV}$ resonance [45, 46] while the region of interest for BBN is between 30 and $400 \mathrm{keV}$. The reaction can be measured at LUNA up to $130 \mathrm{keV}$ (about $400 \mathrm{keV}$ in the laboratory reference system) with the present machine while going to higher energies and eventually overlap with previous direct measurements would require a different, higher voltage, accelerator. A set-up very similar to the one already used for the ${ }^{3} \mathrm{He}\left({ }^{4} \mathrm{He}, \gamma\right){ }^{7} \mathrm{Be}$ reaction [27] could be used since the Q-values of both reactions are very similar. Therefore, a measurement was planned with an intense alpha beam impinging on a windowless gas target filled with ${ }^{2} \mathrm{H}$ followed by the detection of the gammas with a high efficiency and very well shielded HPGe detector in close geometry. However, for this reaction, a side effect exists and has to be dealt with. This consists in the Rutherford scattering of the alpha beam on the deuterium target atoms which can then undergo both ${ }^{2} \mathrm{H}\left({ }^{2} \mathrm{H}, n\right){ }^{3} \mathrm{He}$ and ${ }^{2} \mathrm{H}\left({ }^{2} \mathrm{H}, \mathrm{p}\right)^{3} \mathrm{H}$ reactions. While the latter do not disturb the measurement, the former produce neutrons $(\operatorname{En}(\max )=3.4 \mathrm{MeV})$ which can in principle constitute a problem for the underground laboratory. Moreover, the ${ }^{2} \mathrm{H}\left({ }^{2} \mathrm{H}, \mathrm{n}\right)^{3} \mathrm{He}$ reaction can give rise to $\left(n, n^{\prime} \gamma\right)$ reactions, generating a considerable beam-induced background in the $\gamma$-ray spectrum and, in particular, in the energy region around $1.6 \mathrm{MeV}$ where the capture transition to the ground state of ${ }^{6} \mathrm{Li}$ is expected. The concurring ${ }^{2} \mathrm{H}\left({ }^{2} \mathrm{H}, \mathrm{p}\right)^{3} \mathrm{H}$ process can be used to evaluate the neutron production of the ${ }^{2} \mathrm{H}\left({ }^{2} \mathrm{H}, \mathrm{n}\right)^{3} \mathrm{He}$ reaction: the cross sections of both reactions are well known and from the measured proton rate one can derive the neutron rate. In the last months several tests were performed to study this effect as a function of the target pressure and beam energy. An optimized set-up was developed in order to reduce the target volume and increase the detection efficiency. This allows us to decrease the side effect due to the ${ }^{2} \mathrm{H}\left({ }^{2} \mathrm{H}, \mathrm{n}\right){ }^{3} \mathrm{He}$ reaction decreasing the free path of the scattered ${ }^{2} \mathrm{H}$ nuclei while keeping the expected signal the same. A first data taking of $200 \mathrm{~h}$ was achieved in the best signal-to-noise ratio conditions (experimentally found) of $1 / 10$ in the region of interest (ROI): unfortunately the signature of the ${ }^{2} \mathrm{H}(\alpha, \gamma)^{6} \mathrm{Li}$ reaction is not yet visible. The signal is here evaluated using the recently published astrophysical S-factor obtained at GSI from a Coulomb dissociation experiment [47]. From May until June 2011, 230 hours of data (264 C) have been collected at $400 \mathrm{keV}$ and 236 hours of data $(223 \mathrm{C})$ at $280 \mathrm{keV}$ beam. In this way, since the two ROIs are not overlapping, the signal at $400 \mathrm{keV}$ will be obtained by subtracting the spectrum acquired at $280 \mathrm{keV}$ from the one acquired at $400 \mathrm{keV}$. As expected from the Geant 4 simulation, the shape of both sum spectra is equal with very small deviations, allowing the subtraction of the beam induced background. In order to do this subtraction properly, it is necessary to find the correct normalization factor for the spectrum to be subtracted. The ratio of beam charges is not appropriate, because the beam energies differ too much, and therefore also the neutron production. An analysis method using the whole beam induced background as a neutron "meter", also taking into account the small deviations of the spectrum shape is currently in progress. Final steps to improve the precision of the experimental data are underway or already done: a precise measurement of the detector gamma efficiency along the beam path using not only ${ }^{137} \mathrm{Cs}$ and ${ }^{60} \mathrm{Co}$ but also a weak ${ }^{88} \mathrm{Y}$ source, a check of the gas temperature profile along the beam path, and the calibration of the calorimeter. 


\section{Future}

In the next three-four years, after the completion of the current measurements, the LUNA collaboration will be engaged in the study of a few more $(p, \gamma)$ reactions on Oxygen, Neon and Sodium isotopes. All of them can be studied with the $400 \mathrm{kV}$ accelerator since the relevant energy range, or at least part of it, is accessible to such a machine. After that, reactions that take place at higher temperatures (i.e. higher energies) than those occurring during the hydrogenburning processes studied so far are planned to be measured using an higher voltage machine, in the MV range. For this reason, already in 2007, the LUNA collaboration submitted a Letter of Intent (LoI) [48] to the LNGS Scientific Committee which contained an experimental program mainly devoted to Helium burning reactions. In particular the ${ }^{12} \mathrm{C}(\alpha, \gamma){ }^{16} \mathrm{O}$ reaction, the "Holy Grail" of nuclear astrophysics; the $(\alpha, n)$ reactions on ${ }^{13} \mathrm{C}$ and ${ }^{22} \mathrm{Ne}$, which provide the neutron sources for the s-process (a process responsible for the synthesis of about half of all heavy elements beyond $\mathrm{Fe}$ ); and other $(\alpha, \gamma)$ reactions on ${ }^{14} \mathrm{~N},{ }^{15} \mathrm{~N}$ and ${ }^{18} \mathrm{O}$ relevant for the He-burning stage of stellar evolution are foreseen. The astrophysical relevance of such experimental program is described in the contribution of M. L. Aliotta to this Summer School. After the submission of the LoI, an intense interaction took place between the LUNA collaboration on one side and the LNGS Scientific Committee and the LNGS management on the other side. Two more documents (LoI addendum [49] and LoI update [50]) were submitted by the LUNA collaboration to the LNGS Scientific Committee. A dedicated committee of scientists covering different expertise in the field of nuclear astrophysics was established. This produced a very positive report on the LUNA MV project, strongly recommending its realization at LNGS. The so-called "B node" at LNGS was identified as the best possible place to install the accelerator underground, due to its size and distance from the other LNGS experiments. A detailed technical study of the site preparation including floor sealing, construction of a rough experimental hall etc. is completed. In addition, the LUNA collaboration is investigating how to install a 3.5 MV single ended machine fully equipped inside the B-node. A key issue here is the realization of a neutron shielding to the rest of the laboratory and to the internal rock "walls". In order to evaluate the neutron fluxes potentially produced by the reactions of astrophysical interest, a series of GEANT4 simulations have been developed, assuming a worst-case scenario of $2000 \mathrm{n} / \mathrm{s}$ of $5.6 \mathrm{MeV}$ energy. According to the simulations, an effective shielding can be achieved by using thick concrete doors ( $1 \mathrm{~m}$ frame $+1 \mathrm{~m}$ wall), a $10 \mathrm{~cm}$ HDPE (5\% Li) cover of the rock "walls" and a $20 \mathrm{~cm}$ concrete pavement. The results of the GEANT4 simulations are encouraging since the neutron fluxes just outside the shielding are less than $1 \%$ of the natural LNGS flux. Finally, in February 2011, a two-day Round Table discussion was organized at LNGS with the aim of encouraging new groups to join the LUNA-MV project. Following extensive discussions at the meeting, the constitution of "work packages" is foreseen with the aim of starting a real R\&D phase to address specific aspects of an upgraded facility (these include, for example, the development of neutron detector, gamma detector, gas target, etc.). In the meantime, the request of permission to the competent authorities for installing the MV machine at LNGS will go on. 


\section{References}

[1] C. Rolfs, W. Rodney, Cauldrons in the Cosmos (University of Chicago Press, Chicago, 1988)

[2] C. Iliadis, Nuclear Physics of Stars (John Wiley \& Sons , 2008)

[3] U. Greife et al., NIM A 350 (1994) 327

[4] A. Formicola et al., NIM A 507 (2003) 609

[5] R. Bonetti et al., PRL 82 (1999) 5202

[6] C. Casella et al., NPA 706 (2002) 203

[7] H. Costantini et al., PLB 482 (2000) 43

[8] L. Krauss, B. Chaboyer Science 299 (2003) 65

[9] A. Formicola et al. Phys. Lett. B 591 (2004) 61

[10]G. Imbriani et al. Eur. Phys. J. A 25 (2005) 455

[11] A. Lemut et al. Phys. Lett. B 634 (2006) 483

[12] C. Angulo et al., Nucl. Phys A 656 (1999) 3

[13] U. Schröder et al., Nucl. Phys A 467 (1987) 240

[14] G. Imbriani et al., Astron. Astrophys. 420 (2004) 625

[15] R. C. Runkle et al., Phys. Rev. Lett. 94 (2005) 082503

[16]M. Marta et al., Phys Rev C 78 (2008) 022802(R)

[17]W. Haxton, A. Serenelli Astrophys. J. 687 (2008) 678

[18]B. Aharmin et al. Phys. Rev. C 75 (2007) 045502

[19]J. Hosaka et al. Phys. Rev. D 73 (2006) 112001

[20]G. Bellini et al. arXiv:1104.1816v1 (2011)

[21]E. Adelberger et al. Rev. Mod. Phys. 70 (1998) 055502

[22]A. Caciolli et al. Eur. Phys. J. A 39 (2009) 179

[23]M. Marta et al. Nucl. Instr. and Meth. A 569 (2006) 729

[24]C. Arpesella et al. Appl. Radiat. Isot. 47 (1996) 991

[25]D. Bemmerer et al. Phys. Rev. Lett. 97 (2006) 122502

[26]F. Confortola et al. Phys. Rev. C 75 (2007) 065803

[27]H. Costantini, et al. Nucl. Phys. A 814 (2008) 144

[28]B. S. Sing et al. Phys. Rev. Lett. 93 (2004) 262503

[29]T. A. D. Brown et al Phy.s Rev. C 76 (2007) 055801

[30]A. Di Leva et al., Phys. Rev. Lett 102 (2009) 232502

[31]E. Adelberger et al., Rev. Mod. Phys 83(2011) 195

[32]A. Serenelli et al., arXiv:1104.1639v1 (2011)

[33] J. Knödlseder et al., Astron. Astroph. 345 (1999) 813

[34]C. Winkler et al Astron. Astroph. 411 (2003) L1

[35] T Lee et al., Astrophys. J. Lett 211 (1977) L107

[36]F. Terrasi et al., Nucl. Instr. and Meth. B 259 (2007) 14

[37]B. Limata et al., Phys. Rev. C 82, (2010) 015801

[38]A. Arazi et al., Phys. Rev. C 74, (2006) 025802

[39]D. C. Powell et al., Nucl. Phys. A 660 (1999) 349

[40]F. Strieder et al., Phys. Lett B 707 (2012) 60

[41] C. Iliadis et al., Nucl. Phys A 512 (1990) 509

[42] V. Smith et al., Astrophys. J. 408, 262 (1993)

[43]M. Asplund et al., Astrophys. J. 644, 229 (2006)

[44]A. Coc et al., Astrophys. J. 600, 544 (2004)

[45]R. G. H. Robertson et al, Phys. Rev Lett 47, 1967 (1981)

[46]P. Mohr et al, Phys. Rev. C 50, 1543 (1994)

[47]F. Hammache et al., Phys. Rev C 82, 065803 (2010)

[48]The LUNA Collaboration, LUNA-MV Letter of Intent- LoI 42/07

[49]The LUNA Collaboration, Addendum to the LUNA-MV Letter of Intent- LoI 42/07

[50]A. Guglielmetti, “Update on the LUNA MV Letter of Intent 42/07”, February $26^{\text {th }}, 2010$ 